\title{
Knowledge Surveys in Engineering Statics
}

\section{Mr. Eric Davishahl, Whatcom Community College}

Eric Davishahl is faculty and engineering program coordinator at Whatcom Community College. He previously was faculty and department chair of the engineering department at Everett Community College. Eric has been a member of ASEE since 2001. He currently serves as vice chair of the Pacific Northwest Section and was the recipient of the 2008 Section Outstanding Teaching Award. 


\title{
Knowledge Surveys in Engineering Statics
}

\begin{abstract}
Knowledge surveys ask students to report on their confidence that they can perform specific tasks aligned with course learning outcomes instead of asking students to complete said tasks. This approach allows these surveys to cover a broad range of course topics and cognitive levels while requiring a relatively short amount of time to complete. Administering the surveys multiple times during a course offers a context for students to reflect on their own learning progress and provides useful assessment data to the instructor. Knowledge survey applications documented in the science education literature include their use for both formative and summative assessment of student learning, their use by faculty in assessing the effectiveness of teaching methods, and their use by programs and departments in evaluating curriculum. Reporting on the use of these types of self-assessment surveys; however, is generally absent in the engineering education literature. This paper seeks to introduce this potentially useful tool to the engineering education community by reporting on the development and implementation of a knowledge survey for an engineering statics course. The survey consists of 122 specific success criteria mapped to eight course-level learning outcomes. Students completed the survey three times during the term; once during the first week of class; once before the first exam; and once before the second exam. The paper explores several potential uses of the survey data, examines correlation of the survey responses with exam scores, and concludes that these surveys show promise as a tool for engineering educators. Their effectiveness toward multiple teaching goals warrants further development and investigation.
\end{abstract}

\section{Introduction}

The term "knowledge survey" refers to a specific type of assessment in which students report on their confidence that they can perform specific tasks in lieu of actually completing said tasks. Geoscience educators introduced this idea in the early 2000s and demonstrated its promise as a tool for a variety of assessment purposes and educational goals (Nuhfer, 2003; Wirth, 2005). The process of developing and refining the survey provides a context for instructors to clarify course outcomes and the alignment of associated learning activities. Survey administration early in the term collects formative assessment information for the instructor and sets a baseline for monitoring student learning. The survey items serve as a study guide for course exams and facilitate student self-assessment. End of course survey responses provide summative assessment data to evaluate student progress, new teaching methods, and/or curriculum changes.

Most knowledge surveys ask students to report their confidence level on a three-point scale that generally breaks down as follows:

- 1 point indicates little or no confidence they can complete the task. 
- 2 points indicates at least $50 \%$ confidence.

- 3 points indicates $100 \%$ confidence they can execute the task with accuracy.

This approach allows the survey to cover a broad range of course topics and cognitive levels while requiring a relatively short amount of time of students. Nuhfer and Knipp (2003) provide a useful explanation of the design and potential applications of knowledge surveys. They identify the following survey characteristics as important: the items should clearly frame specific course content; they should cover the full breadth of course outcomes; they should span several levels of the cognitive domain as described by Bloom (1956).

Beyond the geoscience community, Bowers et al (2005) report on their knowledge survey implementation in biology and Bell and Volckman (2011) document use of these surveys in general chemistry. Clauss and Geedley (2010) employed the surveys in biology and math courses in an investigation of how student ability to self-assess might depend on the cognitive level of the task in question. Knowledge survey use has surely spread beyond this handful of reported instances, but the only published mention of their use in an engineering course is at the website for the Boise State University Center for Teaching and Learning Teaching Gallery. Frary (2009) summarizes the basic idea and uses of the surveys and provides an example for a Materials Science course.

This paper aims to introduce this potentially useful tool to the engineering education community by presenting the author's experience developing and implementing a knowledge survey in an engineering statics course. Building on prior work embedding support for student academic skill development in the teaching of statics, the survey includes items specific to metacognitive skill development and learner self-efficacy and is seen as a vehicle for keeping these course goals in front of students throughout the term. The paper investigates a potential correlation of survey responses with exam scores and reflects on the utility and promise of the tool for the various applications described above.

\section{Motivation}

The knowledge survey was developed and implemented as part of a major redesign of the Statics course at Whatcom Community College. The author learned about this type of survey at a STEM education retreat and identified the approach as aligning with multiple goals of the course overhaul. In addition to serving as a tool to assess the effectiveness of new pedagogies, the survey is a vehicle for focusing student attention on the course outcomes and related success criteria independent of the textbook. One goal of the redesign was to shift to the use of open educational resources (OERs) as primary source material for the course. Differences in topic coverage and organization between the two chosen OERs along with subtle differences in language and notation posed a potential challenge for students. A knowledge survey can introduce an umbrella of unifying language and structure in a format that students directly engage with at multiple points in the course.

A second goal of the overhaul was to elevate the student development aspects of the course in importance to the students. Statics' position early in the curriculum for many engineering majors 
results in it being one of the first courses where students must synthesize knowledge gained in prerequisite math and physics coursework and apply it to higher-level analytical problem solving. In addition to the technical skills emphasized in the traditional course content, students' ability to self-assess and regulate their own learning (i.e. metacognition) becomes increasingly important. As Grohs (2015) writes in reference to Statics:

Though the written learning outcomes of a typical undergraduate mechanics course may be exclusively technical, the timing and nature of the course in the overall scheme of an engineering curriculum position it as a course that also requires students to develop proficiency in self-regulation and metacognition.

Several other recent papers note the importance of metacognition and learner self-efficacy in engineering mechanics courses (Boylan-Ashraf 2015; Polo 2015) and examine an assortment of strategies for embedding support for student metacognitive skill development within traditional course content. These interventions include assignments where students write about their problem solving processes (Hanson, 2008; Goldberg, 2015; Goldberg, 2016), exam wrappers (Chen, 2016; Chew, 2016), assignment correction opportunities (Chen 2016), and surveys of study habits (Grohs, 2015).

Inspired by this work and its alignment with an institutional focus on student success, a new course outcome focused on academic skill development was added to the traditional technical outcomes: "Upon successful completion of this course, each student should be able to problem solve in statics by applying analysis strategies, documentation and metacognition." The associated knowledge survey items prompt students to reflect on various aspects of their own academic growth each time they complete the survey.

A third goal for the course was to increase the amount of class time devoted to active learning and hands-on activities using a flipped classroom model. One of the challenges of flipping the classroom is finding effective incentives for students to complete out-of-class preparation. The knowledge survey can serve as a course-long study guide to help direct students to the most important skills and concepts in the reading and support their efforts to self-assess their readiness for class sessions.

\section{Survey Development and Implementation}

The first step in developing the survey was to re-examine and modify the course outcomes in an effort to give each outcome approximately equal weight as measured by the number of supporting survey items. Survey items were developed to support each outcome with a goal of articulating specific tasks that describe a learning progression spanning from the lower cognitive levels of Blooms (i.e. remembering and understanding) to the higher levels (i.e. analysis and synthesis). In total, the survey consists of 122 tasks mapped to eight outcomes and is included in its entirety as an appendix.

The survey was built as a Google form and embedded in a page on the course learning management system (Canvas). This approach facilitates repeated administration outside of class with easy reminders for students via Canvas. Figure 1 shows a screenshot of the survey 
introduction. The instructor assigned the survey on the first day of class and asked students to respond before engaging in the reading and homework assignments. This initial survey was limited to the 57 items covered on exam 1 in hopes of not overwhelming the students.

\title{
ENGR\& 214 Statics Knowledge Survey
}

\author{
The task descriptions below are success criteria associated with the course-level learning outcomes in ENGR\& 214 at \\ Whatcom Community College. \\ For each task, select the response that most closely reflects your current confidence level that you can accurately perform \\ the task. Think about these tasks in the context of solving mechanics problems in PHYS\& 221: Engineering Physics 1. \\ 1. I am unable to perform the task at this time. I am not sure exactly what the task entails. \\ 2. I am able to partially perform the task at this time. I am at least $50 \%$ familiar with the task but could use some more \\ instruction and/or practice.
}

3. I can consistently perform the task for evaluation at this time. I am ready for the test!

Figure 1: Knowledge survey introduction showing instructions to students.

Students completed the survey a second time in week five a few days before the first exam. This timing positioned the second survey just after the due date for the last homework assignment that supported the exam content, but allowed students time between the survey and the exam date to address weaknesses they self-identified using the survey. The second survey included all 122 tasks divided into two sections. The first section was a duplicate of the version from the first day of class. The second section included the remainder of the survey items covered on exam 2 and the final. The third survey occurred during the ninth week of the term prior to the second exam. Students earned 10 participation points as incentive to complete the survey in each instance. This incentive was a very small fraction of the total participation grade in the class.

The instructor talked repeatedly with the class about the motivation for the survey with a goal of increasing student "buy-in" to the learning tool. This effort included a facilitated class

discussion about the concepts of metacognition and learner self-efficacy and their relative importance to success in Statics and other engineering courses. The header material for all homework assignments and in-class activity worksheets included relevant outcomes and success criteria using the same language as the knowledge survey. The goal here was to keep the outcomes language in front of the students continuously. Students were encouraged to use the two pre-exam survey assignments as study guides. The instructor demonstrated how students could trace tasks in the survey back to the relevant homework and/or class activities for review and encouraged them to use this exercise to motivate questions for office hours.

Exams were graded using rubrics constructed from relevant survey items. Figure 2 shows an example problem from the second exam. Table 2 presents the associated grading rubric. The students' first exposure to these rubrics came when they received their graded test. The thinking 
here is that this format of detailed feedback on the first exam might help students make better use of the knowledge survey to support their preparation for future tests.

2. (35 points) The object is to be subjected to the force and couple moment loading shown.

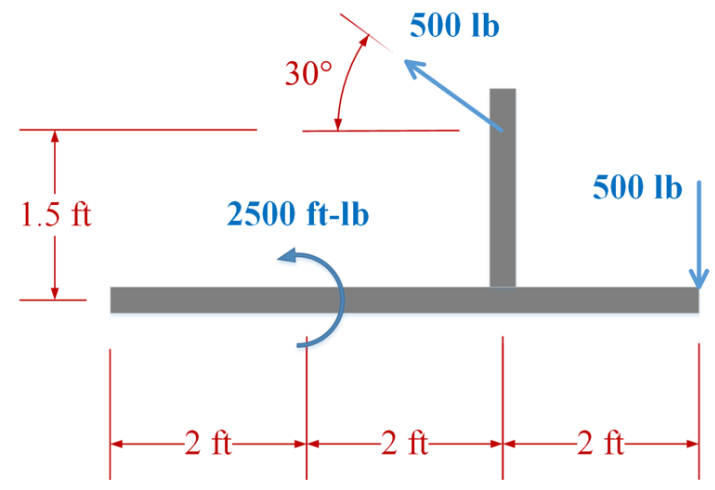

a. (15 pts) Design a support system (choose one or more supports) such that the object is properly supported in equilibrium but not statically indeterminate. Sketch the structural model of the object with its supports in the space below.

b. (20 pts) Calculate the support reactions in your design. Remember to draw the appropriate free-body diagram first.

Figure 2: Sample problem from exam 2.

Table 1: Sample grading rubric implementing selected knowledge survey items.

\begin{tabular}{|l|c|}
\hline Outcome and Success Criteria & Points \\
\hline Problem solve in statics by applying analysis strategies, documentation and metacognition. & \\
\hline $\begin{array}{l}\text { I can (and do) consistently document my problem solving process with clarity, detail, and organization } \\
\text { that others can easily follow. }\end{array}$ & $\mathbf{3}$ \\
\hline $\begin{array}{l}\text { I can apply my math skills (e.g. algebraic manipulation, simultaneous equations, trigonometry, } \\
\text { integration techniques) out of the context of my math classes as needed to implement a solution strategy. }\end{array}$ & $\mathbf{3}$ \\
\hline Analyze relevant forces and moments acting on an object and draw accurate free-body diagrams. & \\
\hline I can draw neat sketches that clearly represent the relevant geometry of an object. & $\mathbf{4}$ \\
\hline I can represent 2-D support models (e.g. pin, roller, fixed) with appropriate reaction forces and couples. & $\mathbf{4}$ \\
\hline $\begin{array}{l}\text { Analyze force systems acting on rigid bodies in equilibrium, including systems involving dry } \\
\text { friction. }\end{array}$ & \\
\hline $\begin{array}{l}\text { I can read forces and moments from a free-body diagram and include them in the appropriate scalar } \\
\text { equilibrium equation. }\end{array}$ & $\mathbf{5}$ \\
\hline I can apply the equations of equilibrium to solve rigid body equilibrium problems in 2-D and 3-D. & $\mathbf{5}$ \\
\hline I can analyze a force system to determine whether it is statically indeterminate. & $\mathbf{3}$ \\
\hline I can analyze a rigid body to determine if it has improper supports. & $\mathbf{3}$ \\
\hline Design structure geometry that meets given performance criteria and constraints. & $\mathbf{5}$ \\
\hline I can design support systems for rigid bodies that will be subjected to multiple loading scenarios. & TOTAL \\
\hline & $\mathbf{3 5}$ \\
\hline
\end{tabular}




\section{Results and Discussion}

Knowledge Survey as a Summative Assessment Tool

One approach commonly used in the literature to evaluate the reliability of the survey data as an indicator of student learning is to test for correlation with exam scores (Nuhfer, 2003; Wirth, 2005; Bowers, 2005; Bell, 2011; Clauss, 2010). Some correlation would seem to be prerequisite for application of the survey response data to other teaching goals such as formative assessment, learner self-efficacy development, and course design evaluation. Another potentially interesting approach would be to compare survey response data to an alternative assessment measure such as the Statics Concept Inventory (Steif, 2005). This potential correlation could be interesting to explore in the future.

It is important to note that in this study, students were encouraged to use the survey as a tool to identify focus areas for their exam preparation efforts. If students successfully followed this advice, then their exam scores should be generally higher than their survey scores. Survey response data was converted to a 100-point scale such that a response of all 3's would yield a normalized score of $100 \%$ and a response of all 1 's would yield a normalized score of $0 \%$ as follows:

$$
\text { NormalizedSurveyScore }=\frac{\text { MeanSurveyResponse }-1}{2}(100 \%)
$$

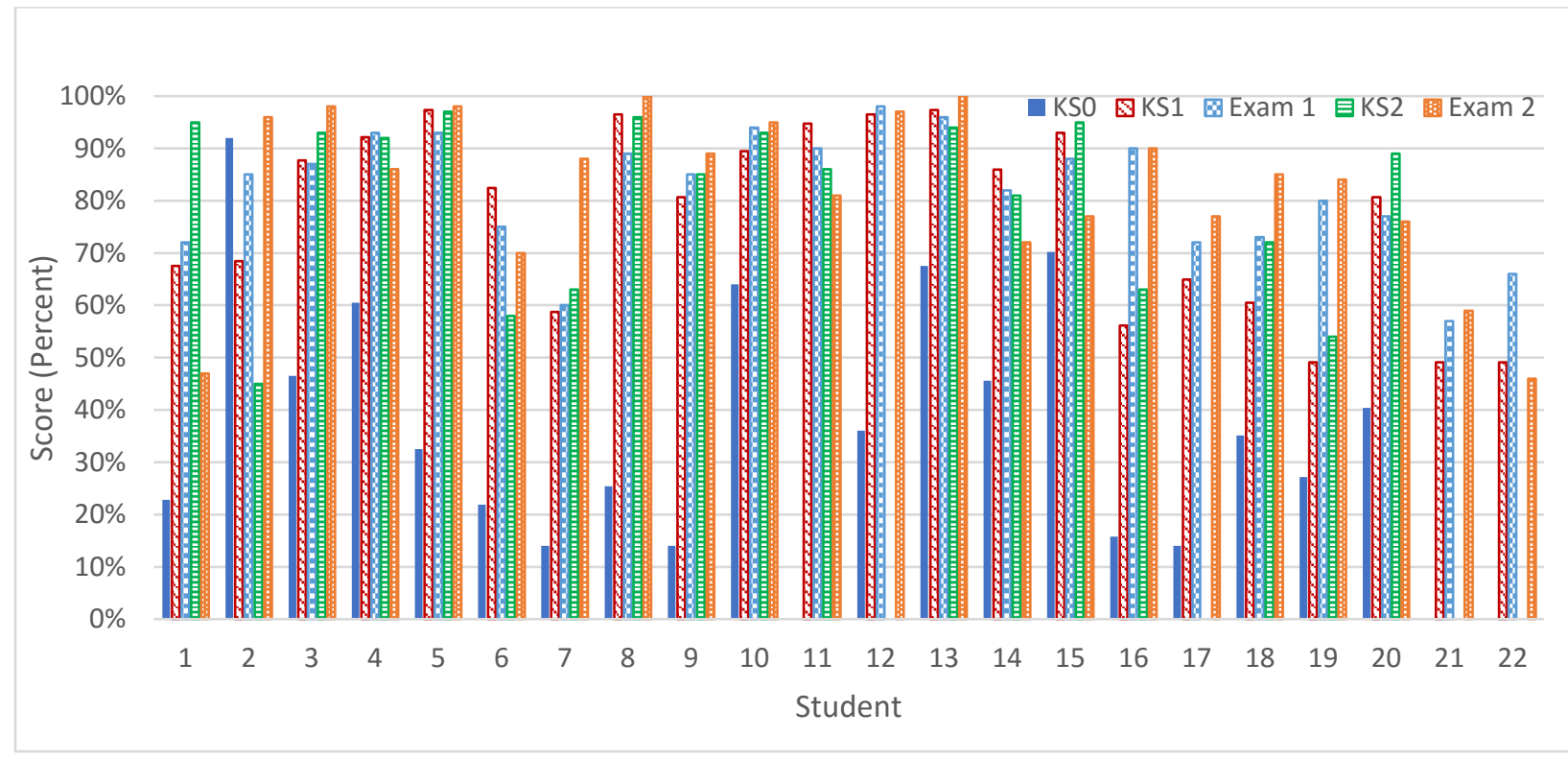

Figure 3: Comparison of survey scores with exam performance. "KSO" refers to the pre-course survey. "KS1" refers to the pre-exam 1 survey. "KS2" refers to the pre-exam 2 survey.

Figure 3 compares student responses to the pre-course survey (KS0 in the figure) with those on the pre-exam surveys (KS1 for pre-exam 1 and KS2 for pre-exam 2) and the respective exam scores. Note student 11 did not respond and students 21 and 22 marked all 1's on the pre-course 
survey, resulting in a normalized score of $0 \%$ for KS0. The KS1 survey had a response rate of $100 \%$ (22 out of 22 students), but only 18 students completed KS2 (response rate of $82 \%$ ). The results show all students except student 2 reporting significant gains in their confidence. Student 2 seems to have been over-confident in the initial survey response as indicated by a significant correction in their responses to KS1.

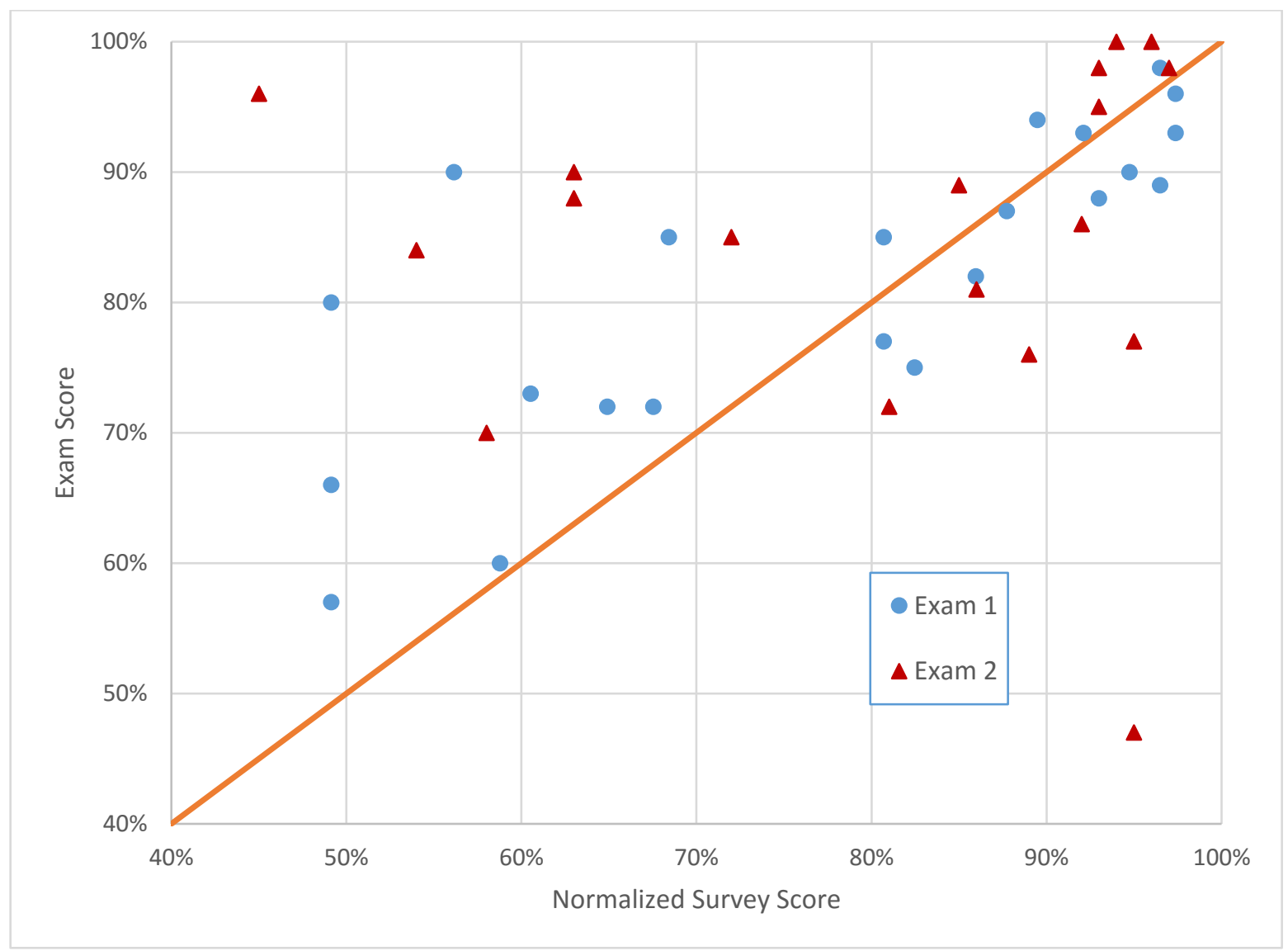

Figure 4: Correlation of exam scores with knowledge survey results. Exam 1 scores plotted versus KS1 results. Exam 2 scores plotted versus KS2 results.

The KS1 scores predict exam 1 results to within $10 \%$ for all but five students $(2,16,18$, 19, and 22). Note that in all five cases these students reported among the lowest confidence on the survey and under-predicted their exam score. There is more variation in the comparison between KS2 and Exam 2. Students 1 and 15 over-predicted their score, while students 2, 7, 16, and 19 under-predicted. Nearly half the students $(3,4,5,8,9,10,11,13$, and 14) predicted their exam scores to within $10 \%$ on both exams.

Figure 4 examines the correlation between the normalized survey scores and exam scores. The correlation coefficient for exam 1 and $\mathrm{KS} 1$ is $r=0.77$, but the correlation for exam 2 is only 0.007. Two outliers explain the weak correlation statistic for exam 2: student 1 scored $47 \%$ on the exam but reported $95 \%$ confidence on the survey; and student 2 did the opposite by scoring $96 \%$ on the exam after reporting $45 \%$ confidence. Disregarding these two data points from the calculation results in a stronger correlation of $r=0.41$. There were also four non-responses to 
the second knowledge survey (students 12, 17, 21, and 22). Three of these four students predicted their exam 1 score to within 10\%. The lower response rate for the KS2 survey may be due to the timing closely coinciding with a project deadline. There may also have been some survey fatigue associated with the third administration of the survey. These two factors might also have negatively affected the time and effort invested by those students who did respond.

Two additional observations are that there is a visibly stronger correlation for the highestperforming students and that the normalized survey score generally under-predicts the exam result for the lowest-performing students. Bell (2005) observed that higher-performing students appeared to be better at estimating their knowledge than lower-performing students and attribute two explaining factors.

1. Low-performing students are generally less capable of self-assessment and exhibit a bias toward overconfidence. This observation was identified by Kruger and Dunning (1999) and has come to be known as the "Dunning-Kruger effect". The bias toward over-confidence; however, does not seem apparent in this study, perhaps with the exception of the dramatic overconfidence expressed by student 1 on the pre-exam 2 survey. The fact that all of these students passed challenging prerequisite courses (Calculus and Physics) could explain the lack of overconfidence bias here. The baseline competence of this student population may be generally higher than in the general chemistry courses in the Bell (2005) study. The opportunity and encouragement students had to address their self-identified weaknesses prior to each exam may also be masking any overconfidence bias.

2. The maximum score of 100 in both the exam and the knowledge survey compresses the top of the scale, potentially enhancing the apparent correlation for the strongest students.

The weaker correlation for lower-performing students may also relate to issues with the survey questions themselves. Wirth (2005) and Nuhfer (2003) both emphasize that the survey should include tasks that span multiple cognitive levels in each content area. This survey includes some instances where the items jump from relatively basic tasks (e.g. "I can identify a structure as a truss by inspecting the geometry and how the load(s) are applied.") to more advanced analytical skills (e.g. "I can analyze a truss using the method of sections."). Adding more intermediate truss analysis tasks such as identifying an appropriate section, applying sign conventions, or drawing free-body diagrams of truss sections would present students with options between indicating basic understanding of a related concept and expressing full confidence in their mastery of this multi-step analysis method. Similar cognitive jumps are evident elsewhere in the survey. These situations may bias the aggregate survey scores of the lower performing students toward under-confidence and can be addressed in future revisions of the survey.

The abstract nature of the survey may also be a factor. The lower-performing students may be less adept at thinking about these tasks without trying to apply them to a specific statics problem. With less understanding of what the task entails, they would be more likely to mark a " 1 " or a " 2 " in their survey response. It could be helpful in future versions to include some example figures to help students contextualize the survey items in their self-assessment. 
Knowledge Survey as Formative Assessment Tool

Reconsidering Figure 3 but focusing on the KS0 results, note that the majority of the responses were a " 1 " indicating little or no student confidence that they could complete the indicated tasks. This comes as no surprise given the timing of the survey, but a few students did signal familiarity with many of the concepts as indicated by a number of "2" responses to several items related to concepts in the prerequisite physics course (e.g. vector analysis, particle equilibrium, and freebody diagrams). It could be interesting to compare these pre-course survey responses with student grades in physics. Perhaps the survey could replace other pre-course assessment tools used to plan review topics to emphasize during class sessions and/or identify students for academic support services such as tutoring.

Knowledge Surveys to Support Learner Skill Development

As discussed above, one of the goals of developing the knowledge survey was for students to use the tool to improve their academic skill and confidence. Several of the survey items under outcome 1 specifically ask students to reflect on their confidence regarding tasks associated with metacognition and learner self-efficacy such as the following:

- I can persist in my effort to solve challenging problems and not give up.

- I can reflect on the effectiveness of my own problem solving strategies and make improvements.

- I can explain my own problem solving strategies to my classmates without simply giving them the answer.

- I can determine when gaps in my own knowledge and/or skills prevent me from solving a problem (i.e. I know when I need to ask for help).

Figure 5 compares KS0 and KS2 results for the tasks associated with outcome 1 (i.e. Problem solve in statics by applying analysis strategies, documentation and metacognition.) and shows most students reporting moderate to substantial confidence growth in this regard. In responding to the survey, students reflected on their cumulative experience with all course activities. There may be some potential here for the combination of the new outcome 1 language and associated survey items to serve as a measure of students' self-efficacy. The degree to which completing the survey itself specifically contributed to this growth could be a subject for further investigation.

The KS0 results in Figure 5 show substantial variation in student confidence regarding their analytical problem solving abilities. In addition to setting a baseline to measure student growth throughout the course, this data could help instructors form diverse collaborative learning teams. These survey items could also identify students for targeted interventions focused on building academic skills.

Students received their normalized survey scores along with their graded exams in hopes they could use this information to calibrate their self-assessment. There is evidence in the data that some students managed to make effective use of the tool in the near consistent result of the lower knowledge survey scores correlating to higher-than-expected exam scores. Were these students successfully remediating their self-observed weaknesses in preparation for the exam? It is 
difficult to know without follow-up interviews with the students. The survey results did inspire office hour visits by students to discuss discrepancies between what they thought they understood and what they were able to demonstrate on the exam. These conversations presented opportunities to explore their approach to the course and to learning in general. It appears anecdotally at least that the knowledge survey was effective at providing students a context for their own metacognition with reminders multiple times throughout the term.

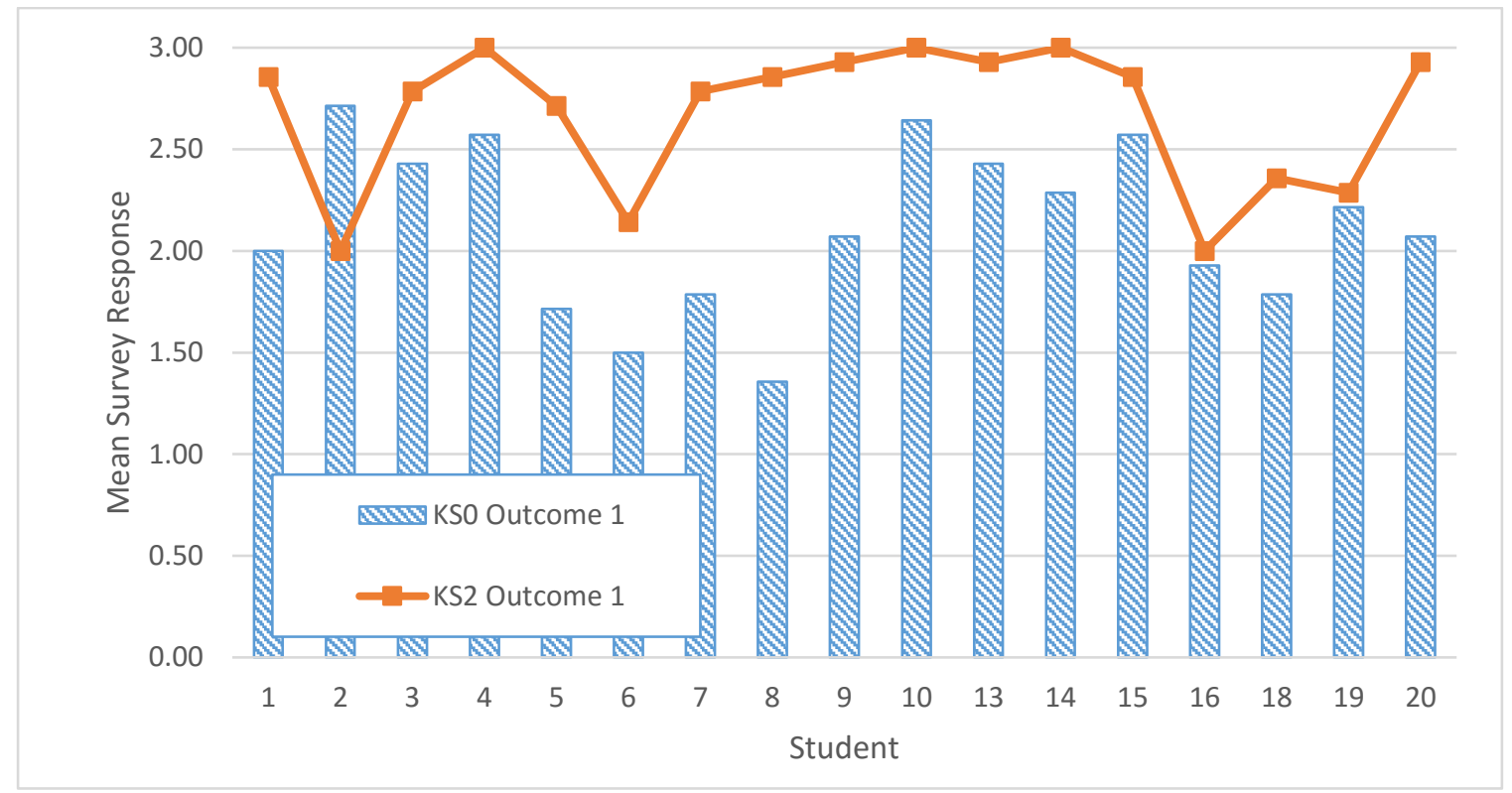

Figure 5: Knowledge survey results for items associated with course outcome 1. Data included only for the 17 students who responded to both the KS0 and KS2 surveys.

An anonymous course feedback survey administered in week 7 included reference to the knowledge survey along with other learning activities with the prompt: "rate [Knowledge Survey] on a scale of 1 (least effective) to 5 (most effective) with regards to how the activity contributes to your learning in this course." The average response was 3.5/5 with no responses of 1 . This feedback indicates that the students found the survey to be valuable for their learning efforts despite its length and repeated use.

\section{Knowledge Survey for Assessment of Course Design}

A conventional textbook served as the organizational anchor for all prior versions of this statics course. As discussed above, one motivation for adopting the knowledge survey was a desire to use multiple OERs as the primary course resource. The survey seemed to provide an effective organizational framework for this approach. Development of the survey items required identification of the specific building blocks and foundational skills leading up to the more complex analytical tasks covered in statics (e.g. frame analysis, shear and moment diagrams). These learning progressions in turn informed decisions about how much material to include in each course reading assignment and the associated class session. Correlating the survey items to homework assignments and in-class activity worksheets provided a metric to align time-on-task requirements for these assignments to their relative importance to the course learning outcomes. 
The survey results provide some valuable data to use along with other assessments to evaluate the effectiveness and content distribution of the learning activities. Consider an example analysis from exam 2, which consisted of three problems: one rigid-body problem (referenced above in Figure 2); a multi-body friction problem; and a frame problem. Of the three problems, students performed poorest on the friction problem. Was this poor performance due to students not mastering the related concepts of body interactions and static friction analysis? Was this poor performance due to issues in the wording or design of the problem? The knowledge survey results provide insight into these questions. Table 3 shows the KS2 survey results for items relating to body interactions and impending motion.

Table 2: Some knowledge survey items relevant to the friction problem on Exam 2 and the associated response data.

\begin{tabular}{|l|c|c|c|c|}
\hline & \multicolumn{3}{|c|}{$\begin{array}{c}\text { Average } \\
\text { Survey Item }\end{array}$} & Response \\
\cline { 3 - 4 } & $\mathbf{1}$ & $\mathbf{2}$ & $\mathbf{3}$ \\
\hline $\begin{array}{l}\text { I can account for the equal and opposite directions of forces between } \\
\text { contacting bodies (Newton's 3rd Law) when drawing multiple free-body } \\
\text { diagrams of interacting bodies. }\end{array}$ & 2.56 & 1 & 6 & 11 \\
\hline $\begin{array}{l}\text { I can analyze a multi-body system to draw the appropriate free-body } \\
\text { diagram(s) that expose specific forces of interest as external }\end{array}$ & 2.56 & 1 & 6 & 11 \\
\hline $\begin{array}{l}\text { I can model the impending slip condition of a contact force using the static } \\
\text { friction coefficient. }\end{array}$ & 2.50 & 1 & 7 & 10 \\
\hline $\begin{array}{l}\text { I can analyze a system involving friction to determine the conditions for } \\
\text { impending motion. }\end{array}$ & 2.67 & 0 & 6 & 12 \\
\hline
\end{tabular}

The overall average response to survey items flagged as relevant to exam 2 was 2.64. All four of these survey items received an average response at or below this average. Approximately one third of the respondents did not indicate confidence they could accurately execute these tasks on an exam. These results correlate well with the class-average exam performance on this problem, thus supporting a conclusion that students could benefit from more learning activities focused on static friction. While one could draw this conclusion based on the exam performance alone, such a conclusion based on a single data point (an exam question) would be vulnerable to issues with the exam question itself.

\section{Conclusion and Future Work}

In summary, this knowledge survey was developed and implemented as part of a substantial overhaul of a statics course as an assessment tool and as an exercise to support student academic skill development in the context of technical course content. Student survey results correlate reasonably well with exam scores and provide valuable assessment data that can inform multiple elements of instructional design. This approach may also help students develop their metacognitive abilities and self-efficacy, but more research could lead to better understanding of the impact. The knowledge survey is attractive in that it can inform multiple aspects of teaching a course with a single learning activity administered outside of class time. 
Future work will focus on exploring ways in which the knowledge survey can support and measure growth in students' academic skill and self-efficacy. This work will likely include development of new knowledge surveys for other courses. There are also plans to continually refine and expand this statics survey to ensure the items span multiple cognitive levels within each content area and place the survey items in the context of specific systems. Development of the exam problem grading rubrics described above has proven particularly informative as a context for this effort. The number of survey items will likely grow in time as language is refined and more intermediate tasks are included. Note that most of the knowledge surveys referenced in the science education literature cited in this paper consist of over 200 items.

\section{References}

Nuhfer, E.B., and Knipp, D. (2003), The knowledge survey: A tool for all reasons. To Improve the Academy, v. 21, p. 59-78.

Wirth, K., and Perkins, D. (2005), Knowledge Surveys: An Indispensable Course Design and Assessment Tool. Innovations in the Scholarship of Teaching and Learning.

https://www.macalester.edu/academics/geology/wirth/wirthperkinsKS.pdf

Bloom, B. S., and Krathwohl, D. R. (1956), Taxonomy of educational objectives: The classification of educational goals / (1st ed.). New York: Longmans, Green.

Bowers, N., Brandon, M., \& Hill, C. D. (2005), The Use of a Knowledge Survey as an Indicator of Student Learning in an Introductory Biology Course. Cell Biology Education, 4(4), 311-322. http://doi.org/10.1187/cbe.04-11-0056

Bell, P., and Volckmann, D. (2011), Knowledge Surveys in General Chemistry: Confidence, Overconfidence, and Performance. Journal of Chemical Education 201188 (11), 1469-1476, DOI: 10.1021/ed100328c

Clauss, J., and Geedley, K., (2010, June), Knowledge Surveys: Students ability to self-assess. Journal of the Scholarship of Teaching and Learning, Vol. 10, No. 2, pp. 14 - 24. http://files.eric.ed.gov/fulltext/EJ890708.pdf

Frary, M. Knowledge Surveys. Center for Teaching and Learning (CTL) Teaching Gallery, Boise State University, 2009. http://scholarworks.boisestate.edu/ctl_teaching/1/

Grohs, J. R., \& Kinoshita, T., \& Novoselich, B. J., \& Knight, D. B. (2015, June), Exploring Learner Engagement and Achievement in Large Undergraduate Engineering Mechanics Courses Paper presented at 2015 ASEE Annual Conference \& Exposition, Seattle, Washington. 10.18260/p.24066

Hanson, J. H. and Williams, J. M. (2008), Using Writing Assignments to Improve Self-Assessment and Communication Skills in an Engineering Statics Course. Journal of Engineering Education, 97: 515-529. doi:10.1002/j.2168-9830.2008.tb00997.x

Chen, J. (2016, June), Effective and Adoptable Metacognitive Tools Paper presented at 2016 ASEE Annual Conference \& Exposition, New Orleans, Louisiana. 10.18260/p.26901

Goldberg, S. R., \& Rich, J. A., \& Masnick, A. M. (2015, June), Efficacy of a Metacognitive Writing-to-Learn Exercise in Improving Student Understanding and Performance in an Engineering Statics Course Paper presented at 2015 ASEE Annual Conference \& Exposition, Seattle, Washington. 10.18260/p.23925 
Goldberg, S. R., \& Rich, J., \& Masnick, A., \& Paretti, M. C., \& Groen, C. J., \& Lutz, B. D., \& McNair, L. D. (2016, June), Examining Students' Metacognitive Awareness Through Analysis of Student-generated Learning Responses Paper presented at 2016 ASEE Annual Conference \& Exposition, New Orleans, Louisiana. $10.18260 /$ p. 26795

Boylan-Ashraf, P. C., \& Billington, S. L., \& Sheppard, S. (2015, June), Using Online and Hands-on Activities to Improve Self-efficacy in Mechanics Paper presented at 2015 ASEE Annual Conference \& Exposition, Seattle, Washington. 10.18260/p.25008

Chew, K. J., \& Chen, H. L., \& Rieken, B., \& Turpin, A., \& Sheppard, S. (2016, June), Improving Students' Learning in Statics Skills: Using Homework and Exam Wrappers to Strengthen Self-regulated Learning Paper presented at 2016 ASEE Annual Conference \& Exposition, New Orleans, Louisiana. 10.18260/p.25633

Polo, F. G. (2015, June), Using the Cognitive Apprenticeship Model to Develop Educational Learning Modules: An Example from Statics Paper presented at 2015 ASEE Annual Conference \& Exposition, Seattle, Washington. $10.18260 /$ p. 25023

Steif, P. S., and Dantzler J. (2005), A Statics Concept Inventory: Development And Psychometric Analysis, Journal of Engineering Education, Vol. 33, pp. 363-371.

Kruger, J., and Dunning, D. (1999), Unskilled and Unaware of It: How Difficulties in Recognizing One's Own Incompetence Leads to Inflated Self-Assessments Journal of Personality and Social Psychology, Vol. 77, No. 6, 1121-1134. 


\section{Appendix (Complete Statics Knowledge Survey Items)}

\section{Problem solve in statics by applying analysis strategies, documentation and metacognition.}

1 I can interpret complex, multi-part problem statements to determine what I am given and what I need to find.

2 I can (and do) consistently document my problem solving process with clarity, detail, and organization that others can easily follow.

3 I can sketch and label clear diagrams to communicate important information about a problem.

4 I can derive a solution to a problem using variables that I assigned to the given quantities BEFORE plugging in numbers and computing a numerical answer.

I can apply my math skills (e.g. algebraic manipulation, simultaneous equations, trigonometry,

5 integration techniques) out of the context of my math classes as needed to implement a solution strategy.

I can synthesize multiple skills and concepts from earlier in this course (Statics) and its

6 prerequisites (Math and Physics) to develop a multi-step strategy for solving a complex problem.

7 I can use order of magnitude analysis to determine if my numerical answers are reasonable.

8 I can use dimensional analysis to evaluate whether my numerical answers are reasonable.

9 I can persist in my effort to solve challenging problems and not give up.

I can reflect on the effectiveness of my own problem solving strategies and make improvements.

I can explain my own problem solving strategies to my classmates without simply giving them the answer.

12 I can determine when gaps in my own knowledge and/or skills prevent me from solving a problem (i.e. I know when I need to ask for help).

13 I can formulate effective questions for class and office hours to address gaps in my own knowledge and skills.

14 I can solve problems on an exam that apply the concepts and methods I have used in class and homework but in different and unfamiliar combinations.

\section{Formulate and solve mechanics problems in two and three dimensions by applying vector analysis techniques.}

15 I can explain why a physical quantity in the field of mechanics is appropriately modeled mathematically as a vector or as a scalar.

16 I can use appropriate notation to indicate which quantities are vectors and which are scalars in a mathematical expression.

I can convert between Cartesian components and magnitude with an angle to represent 2-D vectors as appropriate for mathematical operations and graphical analysis.

18 I can apply vector addition to find the resultant of two or more vectors in two dimensions. I can convert between Cartesian components and magnitude with coordinate angles to represent 3 -D vectors as appropriate for mathematical operations and graphical analysis.

20 I can apply vector addition to find the resultant of two or more 3-D vectors.

21 I can formulate a position vector from given coordinates or dimensional information.

22 I can interpret given dimensional information to determine a unit vector and express it in

22 Cartesian components.

23 I can apply position vectors and unit vectors to determine the direction of the force in a rope or cable in three-dimensional space.

24 I can define and compute the dot product (i.e. scalar product) between two vectors. 
25 I can apply the dot product to find the angle between two vectors in 3-D.

I can apply the dot product and vector addition to resolve a vector into components parallel and perpendicular to a specified structural element.

27 I can define and compute the cross product (i.e. vector product) between two 2-D vectors.

28 I can apply the right-hand rule to determine the direction of the cross product.

29 I can compute the cross product of two 3-D vectors using the determinant. I can select and apply appropriate combinations of vector analysis tools (e.g. vector addition,

30 position vectors, unit vectors, dot products, cross products) to solve complex geometry problems.

\section{Analyze relevant forces and moments acting on an object and draw accurate free-body diagrams.}

31 I can draw neat sketches that clearly represent the relevant geometry of an object.

32 I can replace a body in a system with the normal force and friction force it exerts on adjacent bodies.

33 I can identify and add missing elements to an incomplete free-body diagram.

34 I can determine which dimensions to include on a free-body diagram for use in computing force directions.

35 I can define coordinate systems strategically to simplify the math needed to analyze a force system.

36 I can recognize and ignore internal forces when drawing free-body diagrams. I can analyze a multi-body system to draw the appropriate free-body diagram(s) that expose specific forces of interest as external.

38 I can account for the equal and opposite directions of forces between contacting bodies (Newton's $3^{\text {rd }}$ Law) when drawing multiple free-body diagrams of interacting bodies.

39 I can model the forces exerted by linear springs using Hooke's law.

40 I can determine the direction and sense of tension forces in ropes and cables from given geometry information on a problem figure.

41 I can analyze the presence of pulley(s) in a system to determine changes in the direction of associated tension forces.

I can represent 2-D support models (e.g. pin, roller, fixed) with appropriate reaction forces and couples.

43 I can replace 3-D support models (e.g. bearings, ball and socket, fixed) with appropriate reaction forces and couples.

44 I can identify appropriate support models for real-world structures.

45 I can explain how 2-D and 3-D support models are related.

46 I can determine which dimensions to include on a free-body diagram for use in computing moment arms.

47 I can compute moments due to forces in 2-D systems using a scalar approach.

48 I can compute moments due to forces in 3-D systems using the vector approach (cross product).

49 I can compute the components of moments due to forces in 3-D systems using a scalar approach.

50 I can determine whether a force system is best analyzed in two or three dimensions.

\section{Analyze force systems acting on rigid bodies in equilibrium, including systems involving dry friction. \\ I can reliably solve linear systems of two equations with two unknowns that arise from particle equilibrium problems.}


I can reliably solve linear systems of three equations with three unknowns that arise from rigidbody equilibrium problems or 3-D particle equilibrium problems.

I can reliably solve linear systems of up to six equations with up to six unknowns that arise from 3-D rigid-body equilibrium problems.

I can read forces and moments from a free-body diagram and include them in the appropriate scalar equilibrium equation.

I can apply the equations of equilibrium to solve particle equilibrium problems in 2-D and 3-D.

I can apply the equations of equilibrium to solve rigid body equilibrium problems in 2-D and 3D.

I can analyze two force systems and determine if they are equivalent.

I can compute the couple moment caused by two equal forces acting in opposite directions along parallel lines of action.

I can analyze a system of forces and replace it with an equivalent force acting a specified point and couple moment.

I can model a uniform distributed load with an equivalent force and specify the position at which it acts.

I can model a triangular distributed load with an equivalent force and specify the position at which it acts.

I can model a composite distributed load (consisting of a combination of uniform and triangular loads) with an equivalent force and specify the position at which it acts.

I can analyze a force system to determine whether it is statically indeterminate.

I can identify redundant supports in a statically indeterminate system.

I can analyze a rigid body to determine if it has improper supports.

I can apply equilibrium analysis to determine the direction of a friction force.

I can analyze a system involving friction to determine the conditions for impending motion.

I can model the impending slip condition of a contact force using the static friction coefficient.

I can analyze friction applications such as the use of wedges for mechanical advantage.

I can use friction analysis to determine the line of action for a resultant contact force.

\section{Analyze force interactions in structures (e.g. trusses, frames, and machines).}

71 I can explain the assumptions applied to model a structural element as a two-force member.

72 I can identify two-force members in a structure and replace them with a force of known direction and unknown magnitude.

73 I can identify a structure as a truss by inspecting the geometry and how the load(s) are applied.

74 I can identify zero-force members in a truss.

75 I can explain the reason for including zero-force members in a truss design.

76 I can analyze a truss using the method of joints.

77 I can analyze a truss using the method of sections.

78 I can analyze three-dimensional truss geometries.

79 I can analyze truss geometry to determine stability.

80 I can explain the difference between a frame and a machine in the context of engineering statics. I can decompose a frame/machine into its component parts and draw accurate free-body diagrams of each. I can analyze mechanical connections (e.g. pins, sliders) and model with appropriate forces on a free-body diagram.

83 I can account for equal and opposite force pairs (Newton's 3rd Law) in engineering connections. 
I can be strategic about the order in which I decompose a structure in order to simplify the math needed to compute a specific force.

I can develop and solve the system of scalar equilibrium equations associated with the free-body diagrams of the component parts of a frame or machine.

\section{Analyze and diagram internal forces and moments in structural elements.}

86 I can explain the naming convention for internal resultant loadings.

87 I can apply sign conventions when analyzing internal resultant loadings.

I can section a structural element to expose the internal forces and moments at a point and draw the associated free-body diagram.

I can apply equilibrium analysis to determine the value of the internal axial force at a specified point.

I can apply equilibrium analysis to determine the value of the internal shear force at a specified point.

91 I can apply equilibrium analysis to determine the value of the internal bending moment at a specified point.

92 I can apply equilibrium analysis to determine the shear and bending moment diagrams for a beam subjected to one or more concentrated forces.

93 I can apply equilibrium analysis to determine the shear and bending moment diagrams for a beam subjected to one or more couples.

94 I can apply equilibrium analysis to determine the shear and bending moment diagrams for a beam subjected to one or more distributed loads (uniform, triangular, or composite).

I can apply knowledge of support models to determine the starting and/or ending values of shear force and bending moment.

96 I can apply integration and the relationship between applied load, shear force, and bending moment to determine the shear and bending moment diagrams for a beam.

97 I can analyze changes in the beam loading to generate the shear force and bending moment diagram and/or to check the diagrams that I computed using other methods.

98 I can analyze a shear force and bending moment diagram to identify key points.

99 I can sketch shear force and bending moment diagrams properly aligned under the solved freebody diagram for a beam.

100 I can analyze beams using appropriate and efficient strategies to develop the shear force and bending moment diagrams.

Calculate the centroid, center of mass, and area moment of inertia for planar geometries.

101 I can explain the concept of a centroid of an area.

102 I can explain why the centroid is an important geometric property of areas for mechanics analysis.

103 I can formulate a differential area element for use in computing the centroid or moment of inertia of an area using integration.

104 I can compute the $\mathrm{x}$ and $\mathrm{y}$ coordinates of the centroid of an area using integration.

105 I can retrieve and interpret relevant centroid coordinate formulas from a table of section properties. approach.

107 I can explain the concept of center of mass. 
108 I can compute the center of mass for homogeneous objects with planar geometry.

109 I can explain the concept of area moment of inertia.

110 I can compute the moment of inertia of an area about a specified axis using integration.

111 I can explain the parallel-axis theorem.

112 I can retrieve and interpret relevant moment of inertia formulas from a table of section properties.

113 I can compute the moment of inertia of a composite area about a specified axis using the parallel-axis theorem.

Design structure geometry that meets given performance criteria and constraints.

114 I can specify a single dimension or angle in an object or structure to meet a force magnitude specification.

115 I can analyze a cable support system to determine a range of angle or dimension values that meets a given design constraint.

116 I can design cable and spring support systems to hold an object in a specified position and/or orientation.

117 I can design support systems for rigid bodies that will be subjected to multiple loading scenarios.

118 I can design efficient and stable truss geometry to support a single concentrated load subject to geometry constraints.

119 I can design efficient and stable truss geometry to support multiple loading scenarios.

120 I can specify constraints on applied loads that maintain internal resultant loads at a specified point below specified maximums.

121 I can specify beam loading intensity ranges that maintain internal shear and/or bending moment values below specified maximums.

122 I can modify the geometry of composite areas to achieve a desired centroid location. 